\section{Generalization of Linear Cyclic Pursuit With Application to Rendezvous of Multiple Autonomous Agents}

Arpita Sinha and Debasish Ghose

\begin{abstract}
Cyclic pursuit is a simple distributed control law in which agent $i$ pursues agent $i+1$ modulo $n$. We generalize existing results and show that by selecting the gains of the agents, the point of convergence of these agents can be controlled. The condition for convergence, the range of controller gains and the reachable set where convergence can occur are studied. It is also shown that the sequence in which an agent pursues another does not affect the point of convergence.
\end{abstract}

Index Terms-Author, please supply your own keywords or send a blank e-mail to keywords@ieee.org to receive a list of suggested keywords..

\section{INTRODUCTION}

Multiagent systems are groups of autonomous mobile agents used for automated collaborative operations. Each agent is programmed to sense its environment and make its next move. The challenge in such applications is to design proper control laws such that the agents behave as desired without a centralized controller or global information. Linear cyclic pursuit is one such law that has a simple structure and uses simple local interaction between agents to obtain desired global behavior. This class of control laws are designed to mimic the behavior of biological organisms like dogs, birds, ants, or beetles, and are commonly referred to as the "bugs" problem. Bruckstein et al. [1] modelled the behavior of ants, crickets, and frogs with continuous and discrete pursuit laws and examined the possible evolution of global behavior such as the convergence to a point, collision, limit points, and periodic motion. In [2], Bruckstein describes the stability and convergence of a group of ants in linear and cyclic pursuit. Convergence to a point in linear pursuit is the starting point to the analysis of achievable global formation among a group of autonomous mobile agents [3], [4]. In [3], the evolution of the formation of these agents and the possibility of collision is studied. The stability of both linear and nonlinear pursuit has been studied in [5]. Linear pursuit does not consider any constraint on the dynamics of the agents while nonlinear pursuit imposes nonholonomic or motion constraint on the mobile agents. In [6], role of information flow in the geometric formation of a group of mobile agents with nonholonomic constraints is addressed. In [9]-[12], other methods for multiagent rendezvous are discussed which may include global information and/or communication between agents. A survey of the consensus and agrement problem is presented in [13].

The present work was inspired by the problem addressed in Marshall et al. [5] which considered $n$ identical autonomous mobile agents in linear cyclic pursuit. This system is linear as there are no non-holonomic constraints on the agents. The proof of stability, as available in [5], is restricted to the assumption of equal and positive controller gain for all the agents. Bruckstein et al. [1] briefly mention a case where the controller gain may be inhomogeneous but positive. In the present

Manuscript received August 20, 2005; revised March 17, 2006. Recommended by Associate Editor J. Hespanha. This work was supported in part by by DRDO-IISc Program in Mathematical Engineering.

The authors are with the Department of Aerospace Engineering, Indian Institute of Science, Bangalore 560012, India (e-mail: asinha@ aero.iisc.ernet.in; dghose@aero.iisc.ernet.in).

Digital Object Identifier 10.1109/TAC.2006.883033 note, the controller gains are considered as decision variables that determine the global behavior of the system. The range of values that they can have are obtained from the stability analysis of the system. Some preliminary results on stability of the system were earlier presented in [14]. We show that by selecting the gains suitably, the $n$-agents can be made to meet at some desired point called the rendezvous point. An interesting result in this note is that for any sequence of pursuit which is cyclic, the rendezvous point is the same so long as the initial positions and the controller gains are the same.

\section{Formulation of Linear PuRsuit}

The agents are ordered from 1 to $n$. Agent $i$ pursues agent $i+1$ modulo $n$. The agents start from any arbitrary positions on a plane. The position of the agents at time $t>0$ is given by

$$
Z_{i}(t)=\left[x_{i}(t), y_{i}(t)\right]^{T} \in \mathbb{R}^{2}, \quad i=1,2, \ldots, n .
$$

The kinematic that models agent $i$ 's behavior, is

$$
\dot{Z}_{i}=u_{i}
$$

where $u_{i}$ is the control input given by

$$
u_{i}=k_{i}\left(Z_{i+1}-Z_{i}\right) \quad \forall i .
$$

Thus, the velocity of the $i^{\text {th }}$ agent is proportional to the distance between the $i$ th and the $i+1$ th agent. The controller gain for agent $i$ is $k_{i}$. A special case of the aforementioned pursuit law was considered in [5] where the gains of the agents were assumed to be identical, that is, $k_{i}=k, \forall i$, where $k>0$ is a constant. In the present note the analysis of a more general case is carried out where $k_{i}$ 's can have any value in $\mathbb{R}$ and need not be the same for all the agents. An important point to note here is that $Z_{i}(t)$ need not be restricted to $\mathbb{R}^{2}$. It can, in general, belong to $\mathbb{R}^{m}$. The analysis that follows in the rest of the note will still hold good. When $m=2$, we consider agents that move on a plane, for example, autonomous mobile robots, while $m=3$ would imply movement in three-dimensional (3-D) space which models motion of UAVs in space. Since point mass models of agents are used, collision avoidance is not an issue.

\section{RENDEZVOUS CRITERIA}

The $x_{i}$ and $y_{i}$ coordinates of the $i^{t h}$ agent evolve independently for all $i$. Therefore, they can be decoupled into two identical linear system of equations as

$$
\begin{aligned}
& \dot{X}=A X \\
& A=\left[\begin{array}{cccccc}
-k_{1} & k_{1} & 0 & \cdots & \cdots & 0 \\
0 & -k_{2} & k_{2} & \cdots & \cdots & 0 \\
\vdots & \ddots & & & & \\
\vdots & & \ddots & & & \\
0 & 0 & 0 & \cdots & -k_{n-1} & k_{n-1} \\
k_{n} & 0 & 0 & \cdots & 0 & -k_{n}
\end{array}\right] .
\end{aligned}
$$

The stable formation of the $n$-agent system can be analyzed through a single equation (4) since the equations of motion in the two directions are identical. The stability of (4) depends on the eigenvalues of $A$. If it 


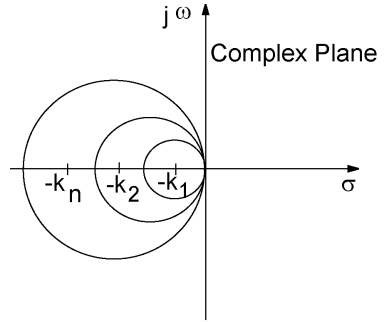

(a)

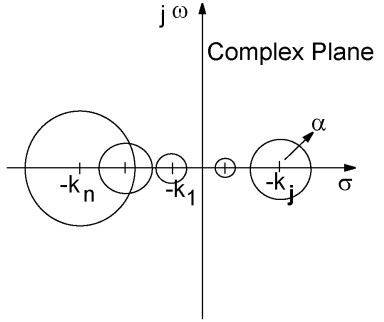

(b)

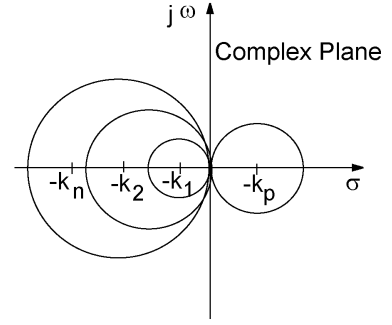

(c)

Fig. 1. (a) Gershgorin discs with all positive controller gains. (b) Gershgorin discs as $\boldsymbol{\alpha}$ changes from 0 to 1. (c) Gershgorin discs with one of the gains negative.

is stable, then the equilibrium point is given by $\dot{X}=0$. This implies that $x_{1}=x_{2}=\cdots=x_{n}=\bar{x}$, that is, all the agents converge to a point $\bar{x}$. Therefore, a rendezvous will occur if $A$ is stable. Note that "rendezvous" in this context just means the convergence of the agents to a single point and does not have the connotation of velocity matching.

We prove stability using Gershgorin's theorem [7]. This result is also stated in [1] but we follow a different route that will help us to generalize the result further.

1) Gershgorin's Theorem [7]: Let $A=\left[a_{i j}\right] \in M_{n}$, and let

$$
R_{i}(A) \equiv \sum_{j=1, j \neq i}^{n}\left|a_{i j}\right|, \quad 1 \leq i \leq n
$$

denote the deleted absolute row sums of $A$. Then, all the eigenvalues of $A$ are located in the union of $n$ discs

$$
\bigcup_{i=1}^{n}\left\{z \in \mathbb{C}:\left|z-a_{i i}\right| \leq R_{i}(A)\right\} \equiv G(A) .
$$

Therefore, if there is a $n \times n$ square matrix $A$, then $n$ circles can be drawn with centers at the diagonal elements of $A$, i.e., $a_{i i}, i=$ $1,2, \ldots, n$, and with radius equal to the sum of the absolute values of the other elements in the same row, that is, $\sum_{j \neq i}\left|a_{i j}\right|$. Such circles are called Gershgorin's discs. All the eigenvalues of $A$ lie in the region formed by the union of all $n$ discs.

\section{CONDITIONS FOR RENDEZVOUS}

Here, we find the range of controller gain for each agent that makes the system stable.

Theorem 1: Consider $n$ mobile agents with kinematics given by (4). For all initial conditions and for $k_{i}>0, \forall i$, all the agents converge to a point.

Proof: Gershgorin's disc theorem is applied to (4). Fig. 1(a) shows the Gershgorin's discs, which lie on the left half of the complex plane for $k_{i}>0, \forall i$. Thus, all eigenvalues of $A$ must lie on the left half plane or at the origin. The characteristic equation of $A$ is

$$
\prod_{i=1}^{n}\left(\lambda+k_{i}\right)-\prod_{i=1}^{n} k_{i}=0 .
$$

From this equation, it is evident that only one eigenvalue lies at the origin provided all gains are positive. Consider a subspace $\mathbb{S}=\{x \in$ $\mathbb{R}^{n} \mid\left[\theta / k_{1} \theta / k_{2} \cdots \theta / k_{n}\right] x=0 ; \theta \neq 0 ; \theta=$ constant $\}$. Following similar arguments as in [5], we can show that $\mathbb{S}$ is $A$-invariant and hence, the trajectories are confined to a $n-1$ dimensional affine subspace which is a translate of $\mathbb{S}$ and contains the initial condition $X(0)$. As in [5], we conclude that we can disregard exactly one zero eigenvalue and determine stability based on the remaining $n-1$ eigenvalues of
$A$. Thus, the linear system (4) is stable and, for any initial condition, the system will converge to the equilibrium point where all the $x_{i}$ 's are equal, which implies that the $n$ agents will finally meet at a point. This can also be proved using the argument (as given in [1]) that, for some constant $\mu, x$ will converge to $\mu \mathbf{e}$ where $\mathbf{e}=\left[\begin{array}{lll}1 & 1 \ldots 1\end{array}\right]^{T}$ is the eigenvector corresponding to the zero eigenvalue .

We will now carry out the stability analysis for the system of $n$-agents, some of whose gains may be negative. Before that, we analyze the characteristic (6) by rewriting it as

$$
p(s)=\prod_{i=1}^{n}\left(s+k_{i}\right)-\prod_{i=1}^{n} k_{i}
$$

Let

$$
f(s)=\prod_{i=1}^{n}\left(s+k_{i}\right) .
$$

Then, $f(s)$ has all real roots located at $-k_{i}, i=1,2, \ldots, n$. If some gains are negative, then some of the roots of $f(s)$ will lie on the righthand side (RHS) of the s-plane.

Lemma 1: If $m$ of the $n$ roots of $f(s)$ are on the RHS of the $s$-plane, then $p(s)$ will have at least $m$ roots on the RHS of the $s$-plane and at the origin, but no roots anywhere else on the imaginary axis.

Proof: Define a matrix $\hat{A}$, obtained from the matrix $A$ in (5), with its nondiagonal entries $k_{i}$ replaced by $\alpha k_{i}, i=1, \ldots, n$, where, $\alpha \in[0,1]$. The characteristics polynomial of $\hat{A}$ is

$$
\prod_{i=1}^{n}\left(s+k_{i}\right)-\prod_{i=1}^{n} \alpha^{n} k_{i} .
$$

When $\alpha=0$, the eigenvalues of $\hat{A}$ are at $-k_{i}, \forall i$, and its characteristic polynomial is $f(s)$. The corresponding Gershgorin's discs are points (circles of zero radius) at $-k_{i}, i=1,2, \ldots, n$. When $\alpha=1, \hat{A}=A$, and the characteristic polynomial is $p(s)$. The Gershgorin's discs, as $\alpha$ varies from 0 to 1 , are shown in Fig. 1(b). Since all the roots remain within the Gershgorin's discs, and from the continuity of the root locus, we observe that the roots do not cross the imaginary axis from either side. At $\alpha=1$, they can at most come to the origin. Since the imaginary axis is not contained in the union of all the Gershgorin's discs, no root will lie on the imaginary axis except at the origin. Therefore, all the $m$ roots of $f(s)$ on the RHS of the s-plane will remain on the RHS, or at the origin. Some roots from left-hand side (LHS) can also migrate to the origin. Hence, at least $m$ roots of $p(s)$ will be on the RHS of the s-plane and at the origin.

Theorem 2: Consider $n$ mobile agents with kinematics given by (4). If two or more agents have negative controller gains, the agents will never converge to a point. 
Proof: This follows directly from Lemma 1. If $m$ agents have negative gains where $m>1$, then $f(s)$ has $m$ roots on the RHS of the $s$-plane. Therefore, according to the previous lemma, at least one root of $p(s)$ will be on the RHS or there will be repeated roots at the origin. In either of the cases the system is unstable. Hence, the agents will not converge to a point.

Now, let us consider the case when only one agent, say $k_{p}$, has negative gain, i.e., $m=1$. In this case, the single root of $f(s)$ on RHS of the s-plane can migrate to the origin and the system represented by $p(s)$ can be stable. Whether this root migrates to the origin or not depends on the magnitude of the gain $k_{p}$. The limit on the maximum negative value that $k_{p}$ can have, such that the system is stable, is given in the following theorem.

Theorem: Consider $n$ mobile agents with kinematics given by (4). The agents will converge to a point if and only if not more than one of the agents (say agent $p$ ) has negative controller gain which is bounded below by $\bar{k}$, i.e., $\bar{k}<k_{p}<0$ and all other agents have $k_{i}>0, \forall i, i \neq$ $p$ where $\bar{k}$ is given by

$$
\bar{k}=-\frac{\prod_{j=1, j \neq p}^{n} k_{j}}{\sum_{i=1, i \neq p}^{n} \prod_{j=1, j \neq i, j \neq p}^{n} k_{j}} .
$$

Proof: From the proof of Theorem 1, one eigenvalue of (4) will be at the origin. When one gain is negative, the Gershgorin discs are as shown in Fig. 1(c). There is one disc on the right half side of the s-plane. However, so long as any of the $(n-1)$ roots of $A$ do not lie in the RHS disc, the system is stable. Consider one agent (say $p^{t h}$ ), whose gain is decreased from a positive value to some negative value. The system will be stable for positive values of the gain (by Theorem 1). At some negative value, the system will become unstable, that is, one of the eigenvalue will cross the imaginary axis and move into the RHS of the s-plane. Since this root has to always remain within the Gershgorin's disc, it must pass through the origin to move from the left to the RHS of the s-plane, as can be seen from Fig. 1(c). Moreover, the system will become unstable at the point where the root reaches the origin. This point gives the bound on the negative gain that the system can have, maintaining its stability. The point at which the root crosses the origin is found from the characteristic equation of $A$. Expanding (6), we get

$$
\lambda\left(\lambda^{n-1}+f_{1}(k) \lambda^{n-2}+f_{2}(k) \lambda^{n-3}+\cdots+f_{n}(k)\right)=0
$$

where, each $f_{j}(k)$ is a function of $k=\left(k_{1}, k_{2}, \ldots, k_{n}\right)$. For a second root to cross the origin,

$$
f_{n}(k)=\sum_{i=1}^{n} \prod_{j=1, j \neq i}^{n} k_{j} \leq 0
$$

from which a bound on $k_{p}$ can be obtained as a function of the other gains, as follows

$$
\left.k_{p}\right|_{f_{n}(k)=0} \leq-\frac{\prod_{j=1, j \neq p}^{n} k_{j}}{\sum_{i=1, i \neq p}^{n} \prod_{j=1, j \neq i, j \neq p}^{n} k_{j}}=\bar{k} .
$$

Thus, for $\bar{k}<k_{p}<0$, all the roots of $A$ are on the LHS of the s-plane and the system is stable. For $k_{p} \leq \bar{k}, f_{n}(k) \leq 0$, and the system is not stable. Thus, the minimum value of $k_{p}$ that ensures stability is bounded by (12) and, so long as $k_{p}>\bar{k}$, the agents will converge to a point. $\square$

Corollary 1: The system of $n$ agents will converge to a point if and only if at most one $k_{i}$ is zero or negative and

$$
f_{n}(k)=\sum_{i=1}^{n} \prod_{j=1, j \neq i}^{n} k_{j}>0
$$

Proof: The proof leads directly from Theorems 1-3.

\section{Rendezvous PoInt or REACHABle PoINT}

We will now show that it is also possible to determine the point of convergence (reachable point) and the possible regions in a plane where rendezvous can occur.

From the proof of Theorem 1, we observe that the property that the trajectories are confined to a $n-1$ dimensional affine subspace is true even when the gains are arbitrary (not necessary positive). Then, we can show (as in [5]) that there exists a linear transformation using a nonsingular matrix $P$, given by

$$
P=\left[\begin{array}{cccccc}
1 & 0 & 0 & \cdots & 0 & 0 \\
0 & 1 & 0 & \cdots & 0 & 0 \\
\vdots & \vdots & \vdots & & & \vdots \\
0 & 0 & 0 & \cdots & 1 & 0 \\
\frac{1}{k_{1}} & \frac{1}{k_{2}} & \frac{1}{k_{3}} & \cdots & \frac{1}{k_{n-1}} & \frac{1}{k_{n}}
\end{array}\right]
$$

such that the new coordinate system is $\hat{X}=P X$ and (4) transforms to (15), as shown at the bottom of the page.

Theorem 4: (Reachable Point) If a system of $n$-agents having their initial positions at $Z_{i 0}=\left[x_{i 0}, y_{i 0}\right]^{T}$ and gains $k_{i}, \forall i$, satisfy Corollary 1 , then they converge to a point $Z_{f}$ given by

$$
Z_{f}=\sum_{i=1}^{n}\left\{\left(\frac{\frac{1}{k_{i}}}{\sum_{j=1}^{n} \frac{1}{k_{j}}}\right) Z_{i 0}\right\}
$$

where $Z_{f}$ is called a reachable point for this system of $n$ agents.

$$
\dot{\hat{X}}=\left[\begin{array}{cccccc|c}
-k_{1} & k_{1} & 0 & \cdots & 0 & 0 & 0 \\
0 & -k_{2} & k_{2} & \cdots & 0 & 0 & 0 \\
\vdots & \vdots & \vdots & & & \vdots & \vdots \\
0 & 0 & 0 & \cdots & -k_{n-2} & k_{n-2} & 0 \\
\frac{-k_{n} k_{n-1}}{k_{1}} & \frac{-k_{n} k_{n-1}}{k_{2}} & \frac{-k_{n} k_{n-1}}{k_{3}} & \cdots & \frac{-k_{n} k_{n-1}}{k_{n-2}} & -\left(k_{n}+k_{n-1}\right) & k_{n} k_{n-1} \\
\hline 0 & 0 & 0 & \cdots & 0 & 0 & 0
\end{array}\right] \widehat{X}
$$


Proof: From (15), we see that $\dot{\hat{x}}_{n}=0 \Rightarrow \hat{x}_{n}=$ constant. Since $\hat{X}=P X$,

$$
\hat{x}_{n}=\frac{1}{k_{1}} x_{1}+\frac{1}{k_{2}} x_{2}+\cdots+\frac{1}{k_{n}} x_{n}=\sum_{i=1}^{n} \frac{x_{i}}{k_{i}}=\text { constant }
$$

where $x_{i}$ is one of the components (say, in the $x$ direction) of the position vectors of the $i^{t h}$ agent. The same equation holds for the components in the $y$ direction. In a vector form

$$
\hat{Z}=\left[\hat{x}_{n}, \hat{y}_{n}\right]^{T}=\frac{Z_{1}}{k_{1}}+\frac{Z_{2}}{k_{2}}+\cdots+\frac{Z_{n}}{k_{n}}=\sum_{i=1}^{n} \frac{Z_{i}}{k_{i}}=\text { constant. }
$$

Since (18) is constant over time, we can write

$$
\sum_{i=1}^{n} \frac{Z_{i 0}}{k_{i}}=\sum_{i=1}^{n} \frac{Z_{i f}}{k_{i}}
$$

where $Z_{i 0}$ and $Z_{i f}$ are the initial and final position of the $i^{\text {th }}$ agent, respectively. Since all the agents converge to a point, $Z_{i f}=Z_{f}, \forall i$. Hence, we get (16).

Let us denote $Z_{f}\left(Z_{i 0}, k\right)$ as the reachable point obtained from the initial point $Z_{i 0}$ and gain $k$ that satisfies Corollary 1 . Then, the set of reachable points (called the reachable set) from the initial point $Z_{i 0}$ is denoted as $\mathcal{Z}_{f}\left(Z_{i 0}\right)$ and is defined as

$$
\mathcal{Z}_{f}\left(Z_{i 0}\right)=\left\{Z_{f}\left(Z_{i 0}, k\right) \mid \forall k \text { satisfying Corollary } 1\right\} .
$$

Thus, for every $\tilde{Z} \in \mathcal{Z}_{f}\left(Z_{i 0}\right)$ there exists a $k$ satisfying Corollary 1 such that $Z_{f}\left(Z_{i 0}, k\right)=\tilde{Z}$.

Thus, the point of convergence of the $n$ agents, given their initial positions, can be controlled by a judicious selection of the gains $k_{i}$. To obtain the region in $\mathbb{R}^{2}$ where a rendezvous of $n$ agents is possible, let us define $S=\left\{Z_{i 0} \mid i=1,2, \ldots, n\right\}$ as a set of $n$ points corresponding to the initial positions of the $n$ agents and let $C o(S)$ be the convex hull of $S$. If $Z_{f} \in C o(S)$, then $Z_{f}$ can be expressed as $Z_{f}=\sum_{i=1}^{n} \lambda_{i} Z_{i 0}$, where $\sum_{i=1}^{n} \lambda_{i}=1$ and $\lambda_{i}>0, \forall i$. If one of the $\lambda_{i}<0$, the set of reachable points expands. This we will show here.

Lemma 2: Consider the set $S=\left\{Z_{i 0} \mid i=1,2, \ldots, n\right\}$. Define a cone $C_{p}$ as finitely generated [8] by the vectors $\left(Z_{p 0}-Z_{i 0}\right), i=$ $1, \ldots, p-1, p+1, \ldots, n$. This cone has vertex at $Z_{p 0}$ and the following hold.

i) If $Z_{p 0} \in \operatorname{Int}\{C o(S)\}$, then $C_{p}$ spans the whole space.

ii) If $Z_{p 0} \in \partial\{C o(S)\}$, then $C_{p}$ is a half space.

iii) If $Z_{p 0} \in V\{C o(S)\}$, then $C_{p}$ is an acute convex cone.

Here, $\partial\{C o(S)\}$ is the boundary of $C o(S)$ and $V\{C o(S)\}$ is the set of vertices of $C o(S)$.

Proof: The proof is straightforward and is omitted.

Theorem 5: Consider a system of $n$ agents with initial positions at $Z_{i 0}, i=1, \ldots, n$. A point $Z$ is reachable if and only if

$$
Z \in C o(S) \bigcup\left\{\bigcup_{p=1}^{n} C_{p}\right\}=\mathcal{P}\left(Z_{i 0}\right)
$$

that is, $\mathcal{Z}_{f}\left(Z_{i 0}\right)=\mathcal{P}\left(Z_{i 0}\right)$.

Proof: If the point $Z$ is reachable then $Z \in \mathcal{Z}_{f}\left(Z_{i 0}\right)$ and so there exists $k$, such that (16) holds. Now, consider the following cases.

Case I: Let $k_{i}>0, \forall i$, then $Z$ is a convex combination of $Z_{i 0}$, $i=1, \ldots, n$. Hence, $Z \in C o(S)$.
Case II: Let one of the gains $k_{p}<0$ and $k_{i}>0, \forall i, i \neq p$. From (19), we can write

$$
\sum_{i=1, i \neq p}^{n} \frac{Z_{i 0}-Z_{p 0}}{k_{i}}=\left(Z-Z_{p 0}\right)\left(\sum_{i=1}^{n} \frac{1}{k_{i}}\right) .
$$

Now, dividing (13) by $\prod_{i=1}^{n} k_{i}$, we get

$$
\sum_{i=1}^{n} \frac{1}{k_{i}}<0
$$

Let $\sum_{i=1}^{n} 1 / k_{i}=-1 / a$ where $a>0$. Then

$$
Z-Z_{p 0}=\sum_{i=1, i \neq p}^{n} \frac{a}{k_{i}}\left(Z_{p 0}-Z_{i 0}\right) .
$$

Thus, $\left(Z-Z_{p 0}\right)$ is a linear combination of the vectors $\left(Z_{p 0}-\right.$ $\left.Z_{i 0}\right), \forall i, i \neq p$ which generate the cone $C_{p}$. Hence, $Z \in C_{p}$. This proves the "only if" (necessary condition) part. To prove the converse, or the sufficiency part, we again consider two cases.

Case I: Let $Z \in C o(S)$. Then, we can find $\alpha_{i}, i=1, \ldots, n$ such that

$$
\sum_{i=1}^{n} \alpha_{i} Z_{i 0}=Z
$$

where, $\sum_{i=1}^{n} \alpha_{i}=1$ with $\alpha_{i} \geq 0, \forall i$. Let us define

$$
k_{i}=\frac{c}{\alpha_{i}}
$$

for all $i$, where $c>0$ is any constant. Thus, $k_{i}>0, \forall i$, and $\sum_{i=1}^{n} 1 / k_{i}=1 / c$.

Replacing $\alpha_{i}$ by $k_{i}$ in (25), we get

$$
Z=\sum_{i=1}^{n}\left\{\frac{\frac{1}{k_{i}}}{\frac{1}{c}}\right\} Z_{i 0}=\sum_{i=1}^{n}\left\{\frac{\frac{1}{k_{i}}}{\sum_{j=1}^{n} \frac{1}{k_{j}}}\right\} Z_{i 0} .
$$

The previous equation is the same as that in (16) and all the gains satisfy Corollary 1 . Therefore, the agents will converge to $Z$. Hence, any $Z \in$ $C o(S)$ is reachable with appropriate positive controller gains.

Case II: Let $Z \in C_{p}$ for some $p$. Then, the vector $Z-Z_{p 0}$ can be expressed as

$$
Z-Z_{p 0}=\sum_{i=1, i \neq p}^{n} \beta_{i}\left(Z_{p 0}-Z_{i 0}\right)
$$

where, $\beta_{i} \geq 0, \forall i$. Therefore,

$$
\begin{aligned}
& Z=\left(\sum_{i=1, i \neq p}^{n}-\beta_{i} Z_{i 0}\right) \\
& \quad+\left(1+\beta_{1}+\cdots+\beta_{p-1}+\beta_{p+1}+\cdots+\beta_{n}\right) Z_{p 0} .
\end{aligned}
$$

Let $1+\beta_{1}+\cdots+\beta_{p-1}+\beta_{p+1}+\cdots+\beta_{n}=-\beta_{p}$ and let us define

$$
k_{i}=\frac{c}{\beta_{i}}
$$




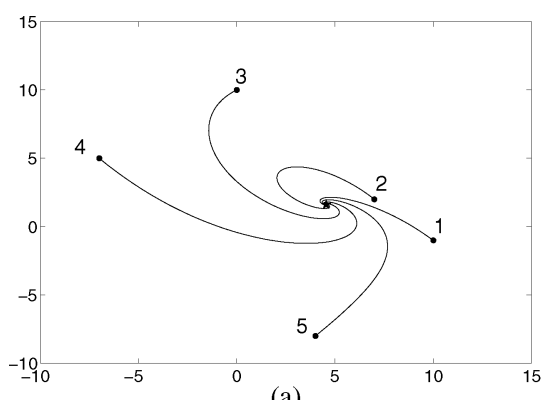

(a)

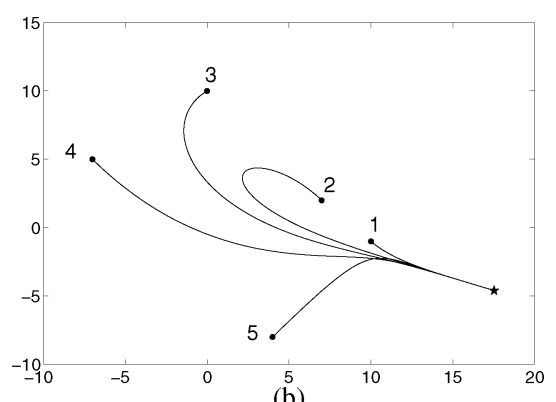

(b)

Fig. 2. (a) Trajectories of five agents with all positive gains. (b) Trajectories of five agents with one gain negative.
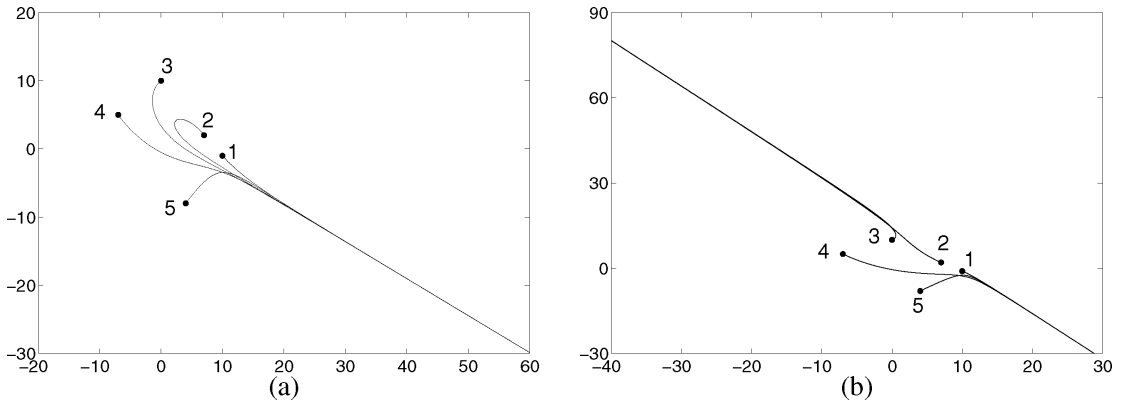

Fig. 3. No convergence cases. (a) Trajectories of five agents. One gain is less than the minimum value required for stability. (b) Trajectories of five agents where two gains $\left(\boldsymbol{k}_{1}\right.$ and $\left.\boldsymbol{k}_{3}\right)$ are negative.

where $c>0$ is a constant. Then, $k_{i} \geq 0, i=1, \ldots, n, i \neq p$ and $k_{p}<0$. To check if $k_{p}>\bar{k}$ from (9), we use (23) and see that

$$
\sum_{i=1}^{n} \beta_{i}=-1 \Rightarrow \sum_{i=1}^{n} \frac{1}{k_{i}}=-\frac{1}{c} .
$$

Since $c>0$, (9) is satisfied. Now, replacing $\beta_{i}$ by $k_{i}$, we get

$$
Z=\sum_{i=1}^{n}\left\{-\frac{\frac{1}{k_{i}}}{\frac{1}{c}}\right\} Z_{i 0}=\sum_{i=1}^{n}\left\{\frac{\frac{1}{k_{i}}}{\sum_{j=1}^{n} \frac{1}{k_{j}}}\right\} Z_{i 0} .
$$

This is the same as (16) and all the gains $k_{i}, \forall i$ satisfy Corollary 1. Hence, any point in $C_{p}$ is reachable. This is true for all $p$. Thus, all the points in $\mathcal{Z}_{f}\left(Z_{i 0}\right)\left(=\mathcal{P}\left(Z_{i 0}\right)\right)$ are reachable.

The set of points $\mathcal{Z}_{f}\left(Z_{i 0}\right)$ forms the reachable set of the system of $n$-agents with the given initial positions. In fact, these agents can be made to converge to any desirable point within this reachable set by suitably selecting the gains. The gains can be selected as given in (26) or (30). We can see that these gains are not unique since none of $\alpha_{i}, \beta_{i}, c$ need to be unique.

All the previous results of this note are proved with the assumption that there is a fixed sequence in which an agent follows another. Now, we show that the sequence in which the cycle is connected does not affect the point of convergence and hence the reachable set.

Corollary 2: (Connection Invariance) Given the system of $n$-agents in cyclic pursuit, the rendezvous point is independent of the sequence of connections between agents.

Proof: Consider (18) which gives the coordinates of the rendezvous point. This equation is independent of the sequence of connection among the agents. The only requirement is that the pursuit should be cyclic. Hence, the rendezvous point is independent of the connectivity of the agents so long as the cyclic pursuit condition is satisfied.

\section{Simulation Results}

A system of five agents are considered. The initial positions of the agents are $S=[(10,-1),(7,2),(0,10),(-7,5),(4,-8)]$. The gains are $k=[4,6,8,10,12]$. The trajectories of agents are shown in Fig. 2(a). The agents converge to $Z_{f}=[4.55,1.6]^{T} \in C o(S)$ which satisfies (16).

Taking the same gains as before for the agents 2 to 5 , we compute the minimum bound for $k_{1}$ from (9) which is $\overline{k_{1}}=-2.86$. Now, assuming the gain of agent 1 as $k_{1}=-1$, which is negative but above the lower bound, the trajectories are shown in Fig. 2(b). The agents converge to a point $Z_{f}=[17.5,-4.6]^{T}$ which satisfies (16). Further $Z_{f} \in C_{1}$.

Assigning a lower value of gain to agent 1 (say $k_{1}=-3$ ), the trajectories of the agents are shown in Fig. 3(a). The agents do not converge to a point and the system is unstable. According to (16), $Z_{f}=[-17.88,12.35]^{T}$ and $Z_{f} \notin \mathcal{Z}_{f}\left(Z_{i 0}\right)$.

When the gains of two agents are negative, i.e., $k_{1}=-1$ and $k_{3}=$ -2 , while all the other gains are the same, the agents do not converge to a point [Fig. 3(b)], illustrating Theorem 2.

We next consider the first case where all the gains are positive and have the same initial positions. Only the order of connection between the agents is changed. The simulation results are shown in Fig. 4(a) and (b) and we find that in both cases the agents converge to the same point. This is the connection invariance property.

Now, suppose we want the agents to converge at the point $Z_{f}=$ $[0,0]^{T}$. We assume that the agents start from the same initial positions that we have considered so far. Then, $[0,0]^{T} \in C o(S)$. One of the convex combination weights on the points of $S$ that yields the desired point $[0,0]^{T}$ is $[0.07,0.08,0.095,0.39,0.37]$. Hence, we can select the gains as $k=c[15.35,12.42,10.50,2.59,2.68]$, where $c>0$ is any constant. Taking $c=1$, the trajectories are shown in Fig. 5(a). The agents converge at the point $[0,0]^{T}$.

To make the agents converge to a point outside $C o(S)$, let us select $Z_{f}=[20,-5]^{T} \notin C o(S)$. However, $Z_{f} \in C_{1}$ and one of the set of $\beta_{i}$ 's that will satisfy $(28)$ is $[-2.16,0.6,0.03,0.43,0.1]$. Assuming $c=1$, the controller gains are $k=[-0.46,1.67,33.33,2.33,10.0]$. 


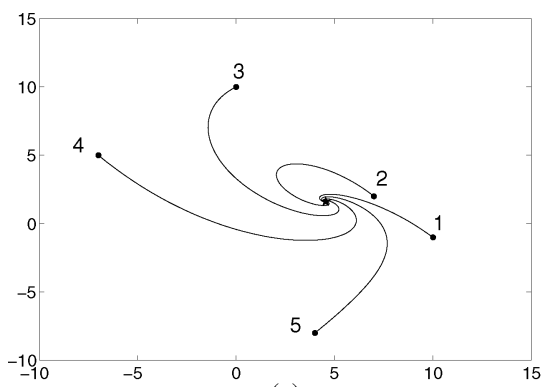

(a)

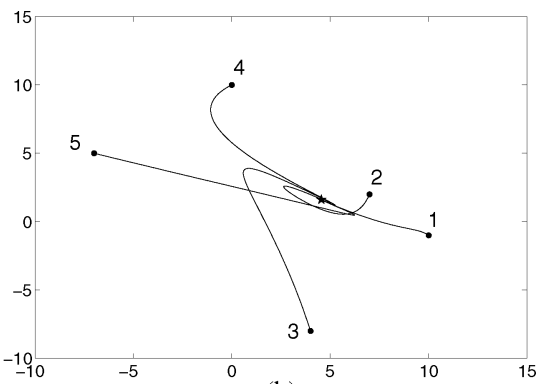

(b)

Fig. 4. Simulation to demonstrate connection invariance of the rendezvous point.

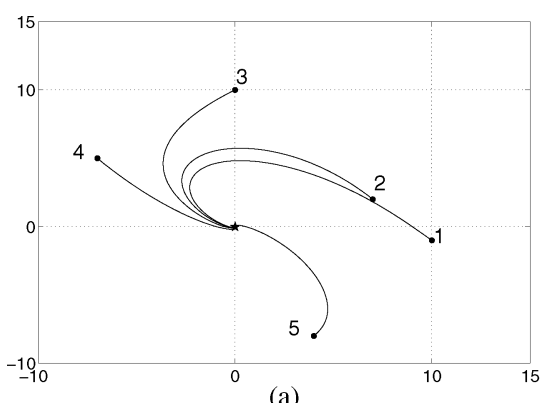

(a)

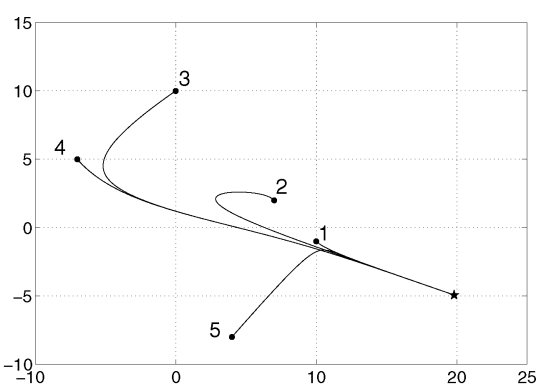

(b)

Fig. 5. Selection of gains for rendezvous at a desired point (a) $\boldsymbol{Z}_{f}=[\mathbf{0}, \mathbf{0}]^{T}$ and (b) $\boldsymbol{Z}_{f}=[\mathbf{2 0}, \mathbf{- 5}]^{T}$.

The trajectories are as shown in Fig. 5(b) and the agents converge to the point $[20,-5]^{T}$.

Thus, the simulation results are in accordance with the analysis given before.

\section{CONCLUSION}

Cyclic pursuit strategies have recently been of much interest among researchers. In this note, several major results are shown. First, generalized convergence properties of linear cyclic pursuit are obtained. In practical applications, homogenous and identical agents may not have exactly the same parameter values. Moreover, these parameter values are sometimes intentionally varied to achieve some other goals. This note obtains the conditions under which the agents using linear cyclic pursuit strategy can have different controller gains and yet converge to a point.

Second, it is shown that the meeting point of the agents can be decided by selecting the gains. The exact reachable set of the points are also obtained. We also show that by making one of the agent's controller gain to be negative, the whole of the state space can be reached in most cases except in some pathological instances (such as when the initial locations of agents are collinear or, in general, restricted to a lower dimensional affine space). A result obtained is that the rendezvous point does not depend on the sequence of connections of the agents so long a cyclic pursuit condition is met.

\section{ACKNOWLEDGMENT}

The authors would like to acknowledge useful comments received from the reviewers and the editor that helped in improving the contents of this note.

\section{REFERENCES}

[1] A. M. Bruckstein, N. Cohen, and A. Efrat, Ants, crickets and frogs in cyclic pursuit Technion-Israel Inst. Technol., Israel Center Intell. Syst. , Haifa, Israel, 1991, Tech. Rep. 9105.
[2] A. Bruckstein, "Why the ant trails loo so straight and nice," Math. Intell., vol. 15, no. 2, pp. 59-62, 1993.

[3] Z. Lin, M. Broucke, and B. Francis, "Local control strategies for groups of mobile autonomous agents," IEEE Trans. Autom. Control, vol. 49, no. 4, pp. 622-629, Apr. 2004.

[4] J. Marshall, Z. Lin, M. Broucke, and B. Francis, V. Kumar, N. Leonard, and A. S. Morse, Eds., "Pursuit strategies for autonomous agents," in Cooperative Control: A Post-Workshop Volume 2003 Block Island Workshop on Cooperative Control, Heidelberg, Germany, 2004, pp. 137-151.

[5] J. A. Marshall, M. E. Broucke, and B. A. Francis, "Formation of vehicles in cyclic pursuit," IEEE Trans. Autom. Control, vol. 49, no. 11, pp. 1963-1974, Nov. 2004.

[6] Z. Lin, B. A. Francis, and M. Maggiore, "Necessary and sufficient graphical conditions for formation control of unicycles," IEEE Trans. Autom. Control, vol. 50, no. 1, pp. 121-127, Jan. 2005.

[7] R. A. Horn and C. R. Johnson, Matrix Analysis. Cambridge, U.K.: Cambridge Univ. Press, 1987.

[8] R. T. Rockafellar, Convex Analysis. Princeton, NJ: Princeton Univ. Press, 1972.

[9] A. Tiwari, J. Fung, J. M. Carson, R. Bhattacharya, and R. M. Murray, "A framework for lyapunov certificates for multi vehicle rendezvous problem," in Proc. American Control Conf., Boston, MA, Jun. 2004, pp. 5582-5587.

[10] C. A. Lua, K. Altenburg, and K. E. Nygard, "Synchronized multi-point attack by autonomous reactive vehicles with simple local communication," in Proc. IEEE Swarm Intelligence Symp., Indianapolis, IN, 2003, pp. $95-102$.

[11] T. McLain and R. Beard, "Cooperative rendezvous of multiple unmanned air vehicles," in Proc. AIAA Guidance, Navigation and Control Conf., Denver, CO, Aug. 2000, AIAA-2000-4369:AAO37126.

[12] J. Lin, A. S. Morse, and B. D. 0 Anderson, "The multi-agent rendezvous problem," in Proc. IEEE Conf. Decision and Control, Dec. 2003, pp. $1508-1513$.

[13] W. Ren, R. W. Beard, and E. M. Atkins, "A survey of consensus problen in multi-agent coordination," in Proc. Amer. Control Conf., Jun. 2004, pp. 1859-1864.

[14] A. Sinha and D. Ghose, "Some generalization of linear cyclic pursuit," in Proc. IEEE India Annu. Conf., Kharagpur, India, Dec. 2004, pp. 210-213. 